\title{
Editorial
}

\section{Digital e sua importância atual no tratamento da insuficiência cardíaca}

Há vários anos, vem sendo discutido o papel do digital no tratamento da insuficiência cardíaca ${ }^{1}$. Embora sua importância clínica nesta síndrome, quando associada a fibrilação atrial, não deixe dúvida, sua eficácia, quando o ritmo é sinusal, constitui, ainda, motivo de controvérsia ${ }^{2-4}$. Esta é, sem dúvida, o resultado de estudos não adequadamente conduzidos e do sucesso das drogas inibidoras da síntese de angiotensina na melhora da qualidade de vida e na longevidade dos pacientes com falência miocárdica 5 .

Os cardiotônicos digitálicos exercem moderado efeito inotrópico positivo no músculo cardíaco, ligando-se às membranas dos cardiomiócitos e inibindo a $\mathrm{Na}+/ \mathrm{k}+\mathrm{ATP}$ ase delas. Atuam, conseqüentemente, aumentando o sódio intracelular, que, por meio do sistema trocador de cálcio, aumenta, também, este e o inotropismo da fibra muscular cardíaca.

A ação inotrópica positiva do digital em dose terapêutica é uma propriedadenão tão importante como se admitia ser no passado. Em pacientes com insuficiência cardíaca, sua ação moduladora neuro-hormonal tem sido valorizada, mais recentemente, einclui redução na atividade plasmática da renina, diminuição do drive simpático, restabelecimento da sensibilidade dos barorreceptores e menor liberação de noradrenalina, com conseqüente diminuição do seu nível plasmático. Este efeito neuro-hormonal permanece durante o tratamento e não é dose-dependente. A combinação das propriedades inotrópica e não-inotrópica produz resultado hemodinâmico benéfico, que se mantém, determinando melhora evidente da fração de ejeção e redução da pressão de enchimento ventricular ${ }^{6}$.

Desde 1980, vêm sendo real izados estudos mais rigorosos e mel hor conduzi dos sobre o uso da di goxina em pacientes com insuficiência cardíaca e ritmo sinusal. As últimas análises destes estudos envolvendo 617 pacientes demonstraram, clínica e estatisticamente, o benefício desta droga ${ }^{7}$, tanto em relação aos sintomas, como na capacidade ao exercício e necessi dade de hospitalização8,9.

Os trials clínicos PROVED ${ }^{10}$ e RADIANCE ${ }^{11}$, com a finalidade de demonstrar a efi cácia e segurança da digoxina em pacientes com insuficiência cardíaca congestiva leve e moderada secundária à disfunção sistólica do ventrículo esquerdo em uso de digoxina e diurético (PROVED), ou digoxina, diurético e inibidor de enzima conversora de angiotensina (RADIANCE), demonstraram que os pacientes que suspenderam o digital, em ambos estudos, apresentaram piora clínica e da função cardíaca, com menor tolerância ao exercício, quando comparados aos pacientes que mantiveram a droga em nível sérico de 0,9 a 2,0mg/mL ).

Até recentemente, o benefício da digoxina na mortalidade por insuficiência cardíaca não havia sido devidamente analisada utilizando-se protocoIos randomizados. O trial DIG (Digitalis Investigators Group) foi recentemente apresentado na Annual Scientific Session of the American College of Cardiology (março/1996); 7.788 pacientes com idade média de 64 anos, com classe funcional II-III (NYHA) e ritmo sinusal, foram acompanhados em 301 centros nos EUA e Canadá; $25 \%$ dos pacientes eram mulheres. $O$ estudo preliminar avaliou os que possuíam disfunção sistólica (fração de ejeção de ventrículo esquerdo menor ou igual a 45\%), embora, aproximadamente, 1.000 pacientes com insuficiência cardíaca e fração de ejeção superior a $45 \%$ fossem seguidos como estudo auxiliar. A dose de digoxina empregada variou de $0,125 \mathrm{mg}$ a $0,5 \mathrm{mg}$ ao dia, baseada no al goritmo que considera idade, sexo, peso e função renal. Os pacientes foram randomizados para receber placebo ou digoxina enquanto mantinham o tratamento com diurético e inibidores de enzima conversora de angiotensina; 93\% que entraram no estudo estavam recebendo esta droga.

Os resultados da primeira análise sobre todas as causas de mortalidade em 37 meses de seguimento revelaram diferença não-significante entre os pacientes tratados com digoxina e com placebo, e que a fração de ejeção baixa e/ou superior a $45 \%$ 
não influenciou no resultado. Eles evidenciaram, contudo, redução estatisticamente significante no risco de morte decorrente da piora da insuficiência cardíaca no grupo tratado com digoxina, comparado com o grupo que recebeu placebo; mas esse benefíci o foi contrabalançado por um risco acumulado de morte atribuído a presumível arritmia ou infarto do miocárdio.

A análise da morbidade, também, demonstrou um aparente benefício da digoxina em pacientes sem arritmia. Os pacientes tratados com a droga tiveram $28 \%$ de redução no risco de primeira hospitalização secundária a piora da insufi ciência cardíaca, comparado com placebo, com redução de risco de infecção pulmonar, presumivel mente indicando menor congestão pulmonar. Houve suspeita de intoxicação digitálica somente em 110 pacientes, e verificou-se que vários pertenciam ao grupo placebo.

Em relação à mortalidade e hospitalização por insuficiência cardíaca, a digoxina mostrou-se eficiente, quando comparada ao placebo.

O estudo posterior de subgrupos sugeriu que seu benefício é maior em paciente com menor fração de ejeção e maior cardiomegalia, e os resultados preliminares deste estudo sugerem que é segura e seu benefício é incrementado com a associação da enzima inibidora da síntese de angiotensina e diurético. Ela é bem tolerada e menos tóxica do que se advoga.

É importante salientar-se que, à luz dos conhecimentos atuais, a digoxina possui ação inotrópica positiva e neuro-hormonal, com efeito benéfico mesmo na insuficiência cardíaca com ritmo sinusal e, até mesmo, quando há predomí- nio da disfunção diastólica do ventrículo esquerdo, havendo dúvidas, ainda, se ela é realmente uma droga pró-arritmica em pacientes com falência miocárdica.

\section{A.C. Lopes}

Editor da Revista da Associação Médica Brasileira; Professor Livre-Docente de Clínica Médica e Chefe da Disciplina de Medicina deUrgência da UniversidadeF ederal deSão Paulo-Escola Paulista de Medicina.

1. Lee C, J ohnson RA, Bingham J B. Heart failure in outpatients. N Engl J Med 1982; 306: 699-705.

2. Fleg J L, Gottlieb SH, Lakatta EG. Is digoxin really important in treatment of congestive heart failure? Am J Med 1982; 73: 244-50.

3. Taggart AJ , J ohnston GD, McDevitt DG. Digoxin withdrawal after cardiac failure in patients with sinus rhythm. J Cardiovasc Pharmacol 1983; 5: 229-34.

4. Young $\mathrm{B}$. Do digital is glycosides still have a role in congestive heart failure? Cardiol Clin 1994; 12: 51-61.

5. The Captopril-Digoxin Multicenter Research Group. Comparative effects of therapy with Captopril and digoxin in patients with mild to moderate heart failure. J AMA 1988; 259: 539-44.

6. The German and Austrian Xamoterol Study Group. Doubleblind placebo-controlled comparison of digoxin and Xamoterol in chronic heart failure. Lancet 1988; 1: 489-93.

7. J aeschke R, Oxman A, Guyatt G. To what extent do congestive heart failure patients in sinus rhythm benefit from digoxin therapy? A systematic overview and meta-analysis. AmJ Med 1990; 88: 279-86.

8. DiBianco R, Shabetai R, Kostuk W et al. A comparison of oral milrinone, digoxin, and their combination in the treatment of patients with chronic heart failure. N Engl J Med 1989; 320: 677-83.

9. Guyatt $\mathrm{GH}$, Sullivan MJ , Fallen EL et al. A controlled trial of digoxin in congestive heart failure. AmJ Cardiol 1988; 61: 371-5.

10. Uretsky BF, Young J B, Shahidi FE et al. Randomized study assessing the effect of digoxin withdrawal in patients with mild to moderate chronic congestive heart failure: results of the PROVED Trial. J Am Coll Cardiol 1993; 22: 955-62.

11. Packer M, Gheorghiade M. Young J B et al. Randomized, double-blind, placebo-controlled, withdrawal study of digoxin in patients with chronic heart failure treated with convertingenzyme inhibitors. N Engl J Med 1993; 329: 1-7. 